Draft Version November 8, 2018

Preprint typeset using $\mathrm{IAT}_{\mathrm{E}} \mathrm{X}$ style emulateapj v. 08/13/06

\title{
EARLY PHASE OBSERVATIONS OF EXTREMELY LUMINOUS TYPE IA SUPERNOVA 2009DC
}

\author{
M. Yamanaka ${ }^{1,2}$, K. S. Kawabata ${ }^{2}$, K. Kinugasa ${ }^{3}$, M. Tanaka ${ }^{4}$, A. Imada ${ }^{5}$, K. Maeda ${ }^{6}$, K. Nomoto $^{6}$, \\ A. Arai $^{1}$, S. Chiyonobu ${ }^{1}$, Y. Fukazawa ${ }^{1}$, O. Hashimoto ${ }^{3}$, S. Honda ${ }^{3}$, Y. Ikejiri ${ }^{1}$, R. Itoh $^{1}$, Y. Kamata $^{9}$, \\ N. Kawai ${ }^{7}$, T. Komatsu ${ }^{1}$, D. Kuroda ${ }^{5}$, H. Miyamoto ${ }^{1}$ S. Miyazaki $^{9}$ O. Nagae ${ }^{1}$, H. Nakaya ${ }^{9}$, \\ T. Ohsugi ${ }^{2}$, T. Omodaka ${ }^{8}$, N. Sakai ${ }^{8}$, M. Sasada ${ }^{1}$, M. Suzuki ${ }^{10}, H$. Taguchi $^{3}, \mathrm{H}^{2}$ Takahashi $^{3}$,

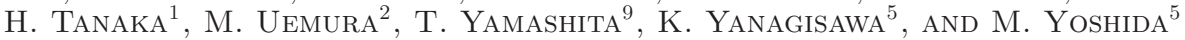

Draft version November 8, 2018

\section{ABSTRACT}

We present early phase observations in optical and near-infrared wavelengths for the extremely luminous Type Ia supernova (SN Ia) 2009dc. The decline rate of the light curve is $\Delta m_{15}(B)=$ $0.65 \pm 0.03$, which is one of the slowest among SNe Ia. The peak $V$-band absolute magnitude is $M_{V}=-19.90 \pm 0.15 \mathrm{mag}$ even if the host extinction is $A_{V}=0$ mag. It reaches $M_{V}=-20.19 \pm 0.19$ mag for the host extinction of $A_{V}=0.29$ mag as inferred from the observed Na I D line absorption in the host. Our $J H K_{s}$-band photometry shows that the SN is one of the most luminous SNe Ia also in near-infrared wavelengths. These results indicate that SN 2009dc belongs to the most luminous class of SNe Ia, like SN 2003fg and SN 2006gz. We estimate the ejected ${ }^{56} \mathrm{Ni}$ mass of $1.2 \pm 0.3 M_{\odot}$ for no host extinction case (or $1.6 \pm 0.4 \mathrm{M}_{\odot}$ for the host extinction of $A_{V}=0.29 \mathrm{mag}$ ). The $\mathrm{C}$ II $\lambda 6580$ absorption line keeps visible until a week after maximum, which diminished in SN 2006gz before its maximum brightness. The line velocity of Si II $\lambda 6355$ is about $8000 \mathrm{~km} \mathrm{~s}^{-1}$ around the maximum, being considerably slower than that of SN 2006gz, while comparable to that of SN 2003fg. The velocity of the C II line is almost comparable to that of the Si II. The presence of the carbon line suggests that thick unburned $\mathrm{C}+\mathrm{O}$ layers remain after the explosion. SN 2009dc is a plausible candidate of the super-Chandrasekhar mass SNe Ia.

Subject headings:

\section{INTRODUCTION}

Type Ia Supernovae (SNe Ia) have been believed to occur when the mass of the progenitor white dwarf (WD) reaches the Chandrasekhar's limiting mass, by mass accretion from a companion star. The homogeneity in their light curves is explained by this scenario and the calibrated luminosity of SNe Ia has been used as an important tool for the constraints on the expansion rate and the dark energy content of the universe (Perlmutter et al. 1999; Riess et al. 1998). However, their progenitors and detailed explosion mechanism have not been confirmed yet (e.g., Nomoto et al. 1997; Hillebrandt \& Niemeyer 2000).

Observationally, SNe Ia have been classified into three

\footnotetext{
${ }^{1}$ Department of Physical Science, Hiroshima University, Kagamiyama 1-3-1, Higashi-Hiroshima 739-8526, Japan; myamanaka@hiroshima-u.ac.jp

${ }^{2}$ Hiroshima Astrophysical Science Center, Hiroshima University, Higashi-Hiroshima, Hiroshima 739-8526, Japan

3 Gunma Astronomical Observatory, Takayama, Gunma 3770702, Japan

${ }^{4}$ Department of Astronomy, School of Science, University of Tokyo, Bunkyo-ku, Tokyo 113-0033, Japan

5 Okayama Astrophysical Observatory, National Astronomical Observatory of Japan, Kamogata, Asakuchi-shi, Okayama 7190232, Japan

${ }^{6}$ Institute for the Physics and Mathematics of the Universe, University of Tokyo, Kashiwa, Japan

${ }^{7}$ Department of Physics, Tokyo Institute of Technology, 2-12-1 Ookayama, Meguro-ku, Tokyo 152-8551, Japan

8 Department of Physics, Faculty of Science, Kagoshima University, 1-21-35 Korimoto, Kagoshima 890-0065, Japan

${ }_{9}$ National Astronomical Observatory of Japan, Osawa, Mitaka, Tokyo 181-8588, Japan

10 Toyota Technical Development, Corp., 1-21 Imae, Hanamotocho, Toyota, Aichi 470-0334, Japan
}

subclasses: normal SNe Ia, overluminous SNe Ia (SN 1991T-like), and faint SNe Ia (SN 1991bg-like) (Branch et al. 1993; Filippenko 1997; Li et al. 2001). The light curves of more luminous SNe Ia decline more slowly (Phillips 1993).

Recently, two extremely luminous SNe Ia 2003fg and 2006gz have been discovered (Howell et al. 2006; Hicken et al. 2007). Their absolute maximum magnitudes are $M_{V}=-19.94 \pm 0.06 \mathrm{mag}$ for SN 2003fg and $-19.74 \pm 0.16$ mag for SN 2006gz, and both SNe show the slowest luminosity evolution. Such an extreme brightness suggests that their progenitor's masses exceed the Chandrasekhar limit ("super-Chandrasekar mass WD") (Howell et al. 2006; Hicken et al. 2007). Interestingly, these SNe showed strong carbon absorptions in their early stages; the $\mathrm{C}$ II $\lambda 6580$ line was seen in SN $2003 \mathrm{fg}$ around maximum, while the line diminished before maximum in SN 2006gz. The expansion velocity inferred from the Si II $\lambda 6355$ line around maximum was slow in SN 2003fg ( $\left.8000 \mathrm{~km} \mathrm{~s}^{-1}\right)$, while it was typical $(\sim 11000-12000 \mathrm{~km}$ $\mathrm{s}^{-1}$ ) in SN 2006gz.

SN 2009dc was discovered on 2009 Apr 9.31 UT at non-filter magnitude of 16.5 near the outer edge of an S0 galaxy UGC 10064 (Puckett et al. 2009, $\mu=34.88 \pm 0.15$ from the $N E D$ database; Falco et al. 1999). A follow-up observation on Apr 16.22 revealed spectroscopic similarity with SN 2006gz before maximum light, including the existence of conspicuous C II features (Harutyunyan et al. 2009). The expansion velocity deduced from the Si II $\lambda 6355$ line is about $8700 \mathrm{~km} \mathrm{~s}^{-1}$, which is slower than that of SN 2006gz (Hicken et al. 2007), but comparable to that of SN 2003fg (Howell et al. 2006).

In this Letter, we show our photometric and spectro- 
scopic observations of this peculiar SN from -8.1 days through +80.5 days after the maximum. The observational results strongly suggest that SN 2009dc is a superChandrasekher SNe Ia having some peculiar properties compared with other candidates.

\section{OBSERVATIONS AND REDUCTION}

We performed $B V R_{c} I_{c}$-band photometry of SN $2009 \mathrm{dc}$ on 30 nights from 2009 Apr 17.8 UT (-8.1 days after the $B$-band maximum; see $\S 3.1)$ through Jul $14.5(+80.5$ days), using HOWPol (Hiroshima One-shot Wide-field Polarimeter; Kawabata et al. 2008) installed to the $1.5 \mathrm{~m}$ KANATA telescope at Higashi-Hiroshima Observatory. The images were reduced according to a standard procedure of a CCD photometry. We performed point-spreadfunction fitting photometry using DAOPHOT package in $I R A F$. The magnitude is calibrated with photometric standard stars in Landolt fields (Landolt 1992) observed on photometric nights. Additionally, we obtained $g^{\prime} R_{c} I_{c}$-band photometric data on 10 nights from -1.7 to +19.3 days, using MITSuME $0.5 \mathrm{~m}$ telescope (Multicolor Imaging Telescopes for Survey and Monstrous Explosions). The MITSuME $R_{c} I_{c}$-band magnitudes are consistent with the KANATA/HOWPol photometry within systematic differences less than 0.03 mag.

Our near-infrared (NIR) photometric data were obtained from -2.8 through +44.0 days using the $1 \mathrm{~m}$ telescope in Kagoshima University and the $1.88 \mathrm{~m}$ telescope at Okayama Astrophysical Observatory of NAOJ equipped with ISLE (near-infrared imager and spectrograph; Yanagisawa et al. 2006). Their magnitude calibrations were performed with nearby stars in 2MASS catalogue.

The optical spectra were obtained using GLOWS (Gunma LOW dispersion Spectrograph) installed to the $1.5 \mathrm{~m}$ telescope at Gunma Astronomical Observatory on six nights from -3.3 through +53.7 days. The wavelength coverage was 4200-8000 $\AA$ and its resolution was $R=\lambda / \Delta \lambda=330$ at $6000 \AA$. The flux was calibrated using several spectrophotometric standard stars taken in the same night. We have removed the strong telluric absorption features from the object spectra using the standard star spectra.

\section{RESULTS AND DISCUSSION}

\subsection{Light Curves}

We show optical and NIR light curves of SN 2009dc in Figure 1 (a). The Galactic extinction of $E(B-V)=$ $0.071 \mathrm{mag}$ and $R_{V}=3.1$ are corrected for (see $\S 3.3$ ). We derive $B$-band maximum magnitude of $15.19 \pm 0.16 \mathrm{mag}$ and its date of $54946.9 \pm 0.2 \mathrm{MJD}($ Apr $25.9 \pm 0.2 \mathrm{UT})$ by polynomial fitting to the light curve. In Figure 1, we compare SN 2009dc with the super-Chandrasekhar SN Ia 2006gz (Hicken et al. 2007; $\Delta m_{15}(B)=0.69$ ), an overluminous SN Ia 1991T (Lira et al. 1998; $\Delta m_{15}(B)=$ 0.99) and a normal SN Ia 2005cf (Wang et al. 2009; $\left.\Delta m_{15}=1.05\right)$.

We notice that the brightness evolution across the maximum is slower than those of SNe $1991 \mathrm{~T}$ and 2005cf in any band. We derive the decline rate of $\Delta m_{15}(B)=$ $0.65 \pm 0.03$ for SN $2009 \mathrm{dc}$, which is similar to $\Delta m_{15}(B)=$ $0.69 \pm 0.04$ of SN 2006gz (Hicken et al. 2007). The decline rate of SN 2009dc is one of the smallest ones among SNe Ia which have ever been published.
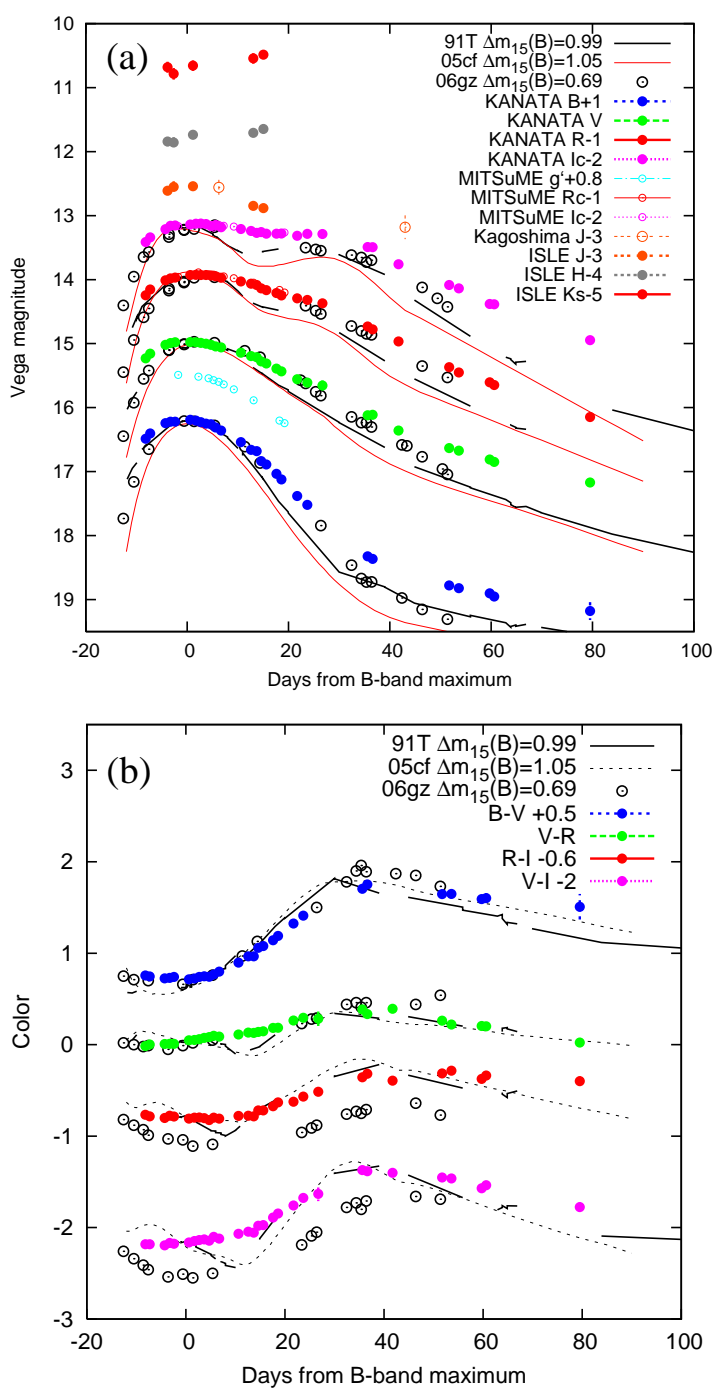

Fig. 1. - (a) $B V R_{c} I_{c^{-}}$-band light curves of SN 2009dc, compared with a super-Chandrasekhar SN Ia 2006gz (Hicken et al. 2007) and an overluminous SN Ia 1991T (Lira et al. 1998) and a normal SN 2005cf (Wang et al. 2009). The Galactic extinction has been corrected for in SN 2009dc. For other SNe the light curves are shifted to match the maximum magnitude. (b) $B-V, V-R c$, $R c-I c, V-I c$ color evolutions, compared with those of $\mathrm{SNe}$ $1991 \mathrm{~T}, 2005 \mathrm{cf}$ and 2006gz. In SN 2009dc, only Galactic extinction $\left(E(B-V)=0.071 \mathrm{mag}\right.$ and $\left.R_{V}=3.1\right)$ is corrected for. In other $\mathrm{SNe}$ Ia, the extinctions in both our and host galaxies are corrected for $(E(B-V)=0.3 \mathrm{mag}$ for SN 1991T, 0.12 for SN 2005cf, 0.12 for SN 2006gz). The color evolution of SN 2009dc is unique compared with those of the other SNe Ia.

The $J$-band light curve (which would be the first NIR light curve of super-Chandrasekhar SNe Ia ever published) merginally shows a more significant dip between the first and second maximum than the I-band light curve. The $H, K$-band light curves suggest the existence of more luminous secondary peak than the first one. These characteristics are likely typical for SNe Ia (Krisciunas et al. 2004; Wang et al. 2009)

\subsection{Color Evolution}

We show the evolution of color indices of SN 2009dc in Figure 1 (b), together with SNe 1991T, 2005cf and $2006 \mathrm{gz}$ for comparison. The evolution of $B-V$ of SN $2009 \mathrm{dc}$ is similar to those of SNe 1991T, 2005cf and 
2006gz; this suggests that the Lira-Phillips relation (homogeneous $B-V$ evolution at 30-90 days, Phillips et al. 1999) also holds for SN 2009dc. We discuss it in §3.3. On the other hand, the evolutions of $V-R, R-I$ and $V-I$ colors in SN 2009dc are somewhat different from those of SNe 1991T and 2005cf; the color indices of SN 2009dc become redder monotonously, while the other SNe Ia (except for SN 2006gz) have small troughs at 1015 days after the $B$-band maximum. SN 2006gz shows the color evolution similar to SN 2009dc, while it keeps bluer at -8 through +60 days and shows broad troughs in the $R-I$ and $V-I$ curves.

\subsection{Host Galactic Extinction and Absolute Magnitude}

To confirm that SN 2009dc is one of the most luminous $\mathrm{SNe}$ Ia, it is important to determine the extinction toward this SN (Galactic + host).

The Galactic color excess is estimated to be $E(B-$ $V)=0.071 \mathrm{mag}$ (Schlegel et al. 1998), corresponding to an extinction of $A_{V}=0.22 \mathrm{mag}$ within our Galaxy (a typical selective extinction $R_{V}=3.1$ is assumed). On the other hand, the extinction within the host galaxy is somewhat uncertain. If the Lira-Phillips relation holds for SN $2009 \mathrm{dc}$, it predicts a reddening of $E(B-V)=(0.37 \pm 0.08)$ mag. However, this is likely an overestimation because the equivalent width $(\mathrm{EW})$ of the Na I D absorption line in the host galaxy $(1.0 \AA)$ is only twice the EW of Na I D in our Galaxy ( $0.5 \AA$; Tanaka et al. 2009). If we assume that the extinction is simply proportional to the EW, $E(B-V)=0.14$ mag is plausible for the host extinction. Additionally, the empirical relation between the color excess and the EW of $\mathrm{Na}$ I D (Turatto et al. 2003; we adopt their lower extinction case) predicts $E(B-V)=0.15$ mag. These two values are consistent. This also suggests that the relation is consistent with our Galaxy's values of $E(B-V)=0.071$ mag and the $\mathrm{EW}$ of $=0.5 \AA$ for the Galactic Na I D line. In Table 1 we summarize the estimated absolute magnitude for various cases of extinction parameters. There is an additional uncertainty for the extinction due to the diversity of $R_{V}$ (Wang et al. 2006; Krisciunas et al. 2006; Elias-Rosa et al. 2006). We adopt $R_{V}=2.1$ and 3.1 following Hicken et al. (2006). Even if $E(B-V)=0 \mathrm{mag}$ within the host galaxy is assumed, the absolute maximum magnitude is $M_{V}=-19.90 \pm 0.15 \mathrm{mag}$, indicating that SN $2009 \mathrm{dc}$ is one of the most luminous SNe Ia.

Krisciunas et al. (2004) pointed that the absolute maximum magnitude in NIR bands does not depend on the decline rate (except for faint $\mathrm{SNe} \mathrm{Ia}$ ) and derived the mean values of $-18.6 \mathrm{mag},-18.2 \mathrm{mag}$ and -18.4 mag for $J, H$ and $K s$-bands, respectively. We estimate the absolute maximum magnitudes of SN 2009dc as $M_{J}=-19.20 \pm 0.16 \mathrm{mag}, M_{H}=-19.00 \pm 0.17 \mathrm{mag}$, $M_{K s}=-19.19 \pm 0.17 \mathrm{mag}$ for zero host extinction case, which suggests that SN 2009dc is very luminous even in NIR wavelengths.

It is interesting to examine whether the maximum magnitude- $\Delta m_{15}(B)$ relation (e.g. Altavilla et al. 2004) holds for this brightest SN Ia. If there is no host extinction, the absolute $\mathrm{V}$ magnitude derived from observations is roughly consistent $(<1-2 \sigma)$ with the relation. On the other hand, it seems much brightter (by $3-4 \sigma$ ) than the prediction of this relation if the host extinction is

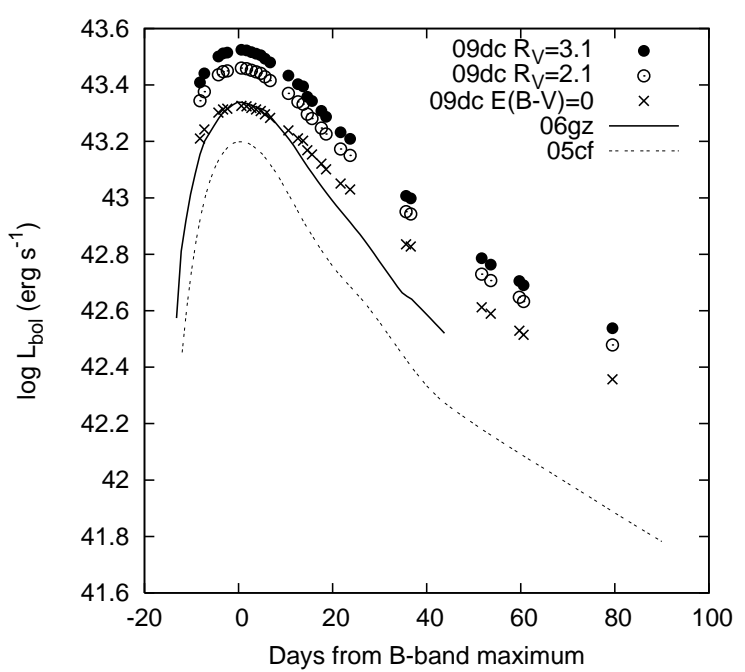

FIG. 2.- Bolometric light curve of SN 2009dc. Filled and open circles show the cases of the host extinction $E(B-V)=0.14$ mag with $R_{V}=3.1$ and 2.1 , respectively The asterisk shows the case with no host extinction. The bolometric light curves of the normal SN Ia 2005cf (dashed line; Wang et al. 2009) and the superChandra SN 2006gz (thick line; Hicken et al. 2007) are shown for comparison.

$A_{V}=0.29 \mathrm{mag}$.

\subsection{Bolometric Light Curve and ${ }^{56} \mathrm{Ni}$ Mass}

We obtain the bolometric luminosity of SN $2009 \mathrm{dc}$ using our $B V R_{c} I_{c}$-band data, assuming that the optical luminosity occupies about $60 \%$ of the bolometric one around maximum brightness (Wang et al. 2009). Because of the uncertainty involved in this assumption, we consider that this bolometric luminosity may have a somewhat large systematic error $(\sim 20 \%)$. To confirm the reliability, we also calculate the bolometric luminosity from $B V R I+J H K s$ data at -3 days and check the consistency. We assume that the integrated $B V R I J H K s$ luminosity is $80 \%$ of the total (Wang et al. 2009). They agree within an error of $\sim 12 \%$. But this discrepancy includes an uncertainty of determining the maximum date of $J H K s$-band luminosity.

The obtained bolometric light curves are shown in Figure 2. We also calculate the bolometric luminosity of SN $2005 \mathrm{cf}$ with the same assumption and confirm the consistency with the results by Wang et al. (2009). Even if we assume that the host extinction is zero, the maximum bolometric luminosity is $L_{\max }=(2.1 \pm 0.5) \times$ $10^{43} \mathrm{erg} \mathrm{s}^{-1}$, which is comparable to that of SN $2006 \mathrm{gz}$, $(2.18 \pm 0.39) \times 10^{43} \mathrm{erg} \mathrm{s}^{-1}$ for $E(B-V)=0.18 \mathrm{mag}$ (Hicken et al. 2007). When we adopt the host extinction of $E(B-V)=0.14 \mathrm{mag}$ and $R_{V}=3.1$, $L_{\max }=(3.3 \pm 0.9) \times 10^{43} \mathrm{erg} \mathrm{s}^{-1}$, which is likely to exceed that of SN 2003fg $\left(\sim(2.5-2.8) \times 10^{43} \mathrm{erg} \mathrm{s}^{-1}\right.$; Howell et al. 2006).

The mass of ejected ${ }^{56} \mathrm{Ni}$ can be roughly estimated from the peak luminosity (e.g., Arnett 1982). Stritzinger \& Leibudgut (2005) suggested that the ${ }^{56} \mathrm{Ni}$ mass depends approximately on the peak bolometric luminosity and its rising time ( $t_{r}$ days), as

$$
L_{\max }=\left(6.45 e^{\frac{-t_{r}}{8.8 \mathrm{~d}}}+1.45 e^{\frac{-t_{r}}{111.3 \mathrm{~d}}}\right)\left(\frac{M_{\mathrm{Ni}}}{M_{\odot}}\right) \times 10^{43} \mathrm{erg} \mathrm{s}^{-1} .
$$


TABLE 1

Estimated MAXimum Absolute magnitude, Luminosity AND THE ${ }^{56}$ Ni mass in some EXTINCTION CASES

\begin{tabular}{ccccccc}
\hline $\begin{array}{l}\mathrm{E}(B-V) \\
\text { Galactic }\end{array}$ & $\begin{array}{c}\text { host } \\
\text { hag }\end{array}$ & $\begin{array}{c}\text { host } A_{V} \\
(\mathrm{mag})\end{array}$ & $R_{V}$ & $\begin{array}{c}M_{V, \max } \\
(\mathrm{mag})\end{array}$ & $\begin{array}{c}L_{\max } \\
\left(\mathrm{erg} \mathrm{s}^{-1}\right)\end{array}$ & $\begin{array}{c}56 \mathrm{Ni} \text { mass } \\
\left(M_{\odot}\right)\end{array}$ \\
\hline \hline 0.07 & 0 & - & 3.1 & $-19.90 \pm 0.15$ & $(2.1 \pm 0.5) \times 10^{43}$ & $1.2 \pm 0.3$ \\
0.07 & 0.14 & 0.29 & 2.1 & $-20.19 \pm 0.19$ & $(2.9 \pm 0.8) \times 10^{43}$ & $1.6 \pm 0.4$ \\
0.07 & 0.14 & 0.43 & 3.1 & $-20.32 \pm 0.19$ & $(3.3 \pm 0.9) \times 10^{43}$ & $1.8 \pm 0.5$ \\
\hline
\end{tabular}

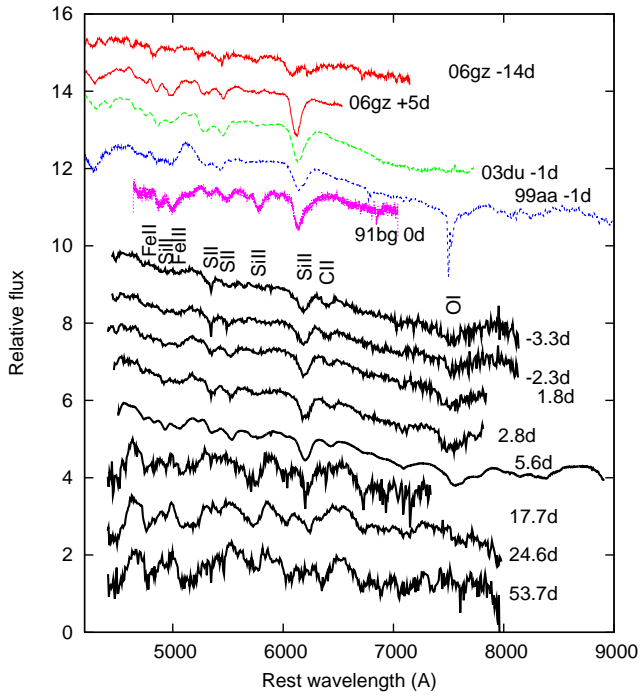

FIG. 3.- Spectra of SN 2009dc compared with those of other SNe Ia; subluminous object SN 1991bg, over-luminous object SN 1999aa, typical object SN 2003du and the super-Chandrasekhar SN Ia 2006gz. All spectra are calibrated to the Rest frame wavelength. The spectrum of SN $2009 \mathrm{dc}$ at +5.5 days is from Tanaka et al. (2009). The telluric absorption feature have been removed.

The slow evolution of brightness in SN 2009dc around the maximum suggests that the rising time of the bolometric luminosity is comparable to or slightly longer than those of SN 2006gz ( 18.5 days; Hicken et al. 2007) or typical SNe Ia ( 19 days; e.g., Conley et al. 2006). Assuming $t_{r}=20$ days for SN 2009dc, we derive the ${ }^{56} \mathrm{Ni}$ mass of $1.2 \pm 0.3 M_{\odot}$ for no host extinction case. It reaches $1.8 \pm 0.5 M_{\odot}$ if we assume the host extinction of $E(B-V)=0.14 \mathrm{mag}$ and $R_{V}=3.1$ (Table 1). Although the derived $L_{\max }$ and the ${ }^{56} \mathrm{Ni}$ mass still include somewhat large uncertainties, the observational results suggest that the mass of the progenitor might exceed the Chandrasekhar-limit.

\subsection{Spectral Evolution}

In Figure 3, we compare the spectra of SN 2009dc with those of the super-Chandrasekhar SN 2006gz at -14 days and +5 days (Hicken et al. 2007), the subluminous SN 1991bg (Filippenko et al.1992), the overluminous SN 1999aa (Garavini et al.2004) and the typical SN 2003du (Stanishev et al.2007) around maximum. The spectra of SN 2009dc around maximum show Si II $\lambda 6355$ absorption, a W-shape S II absorption feature and Fe-group multiple absorptions. Additionally, the absorption line of C II $\lambda 6580$ is seen in SN 2009dc, even a few days after the maximum. This feature is seen only in a small fraction of SNe Ia at their earliest epochs (Tanaka et al. 2008). In the super-Chandrasekhar candidate SN 2006gz, the

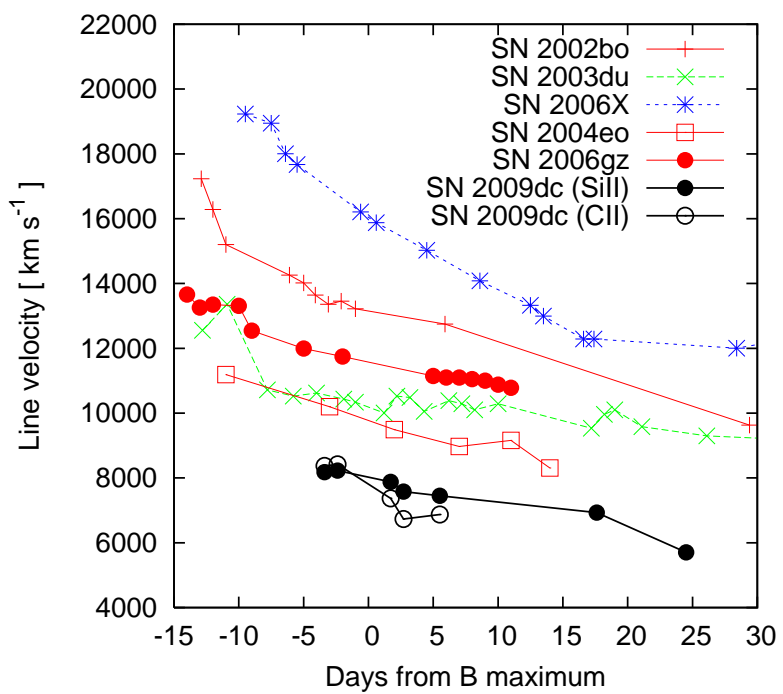

FIG. 4.- Si II $\lambda 6355$ line velocity evolution of SN 2009dc and comparative SNe Ia, 2006gz (Hicken et al. 2007), 2006X (Yamanaka et al. 2009), 2004eo (Pastorello et al.2007), 2003du (Stanishev et al.2007), 2002bo (Benetti et al.2004). We also show the C II $\lambda 6580$ line velocity of SN 2009dc with black open circles. The low expansion velocity of SN 2009dc is remarkable.

carbon feature also exists, while the feature is significant only in the earliest stages $(\lesssim-10$ days; Hicken et al. 2007). In another, more distant super-Chandrasekhar $\mathrm{SN} 2003 \mathrm{fg}$, the C II $\lambda 6580$ feature is not significant at +2 days, while there is a possible carbon feature around $4150 \AA$ at the same epoch. These indicate that a massive $\mathrm{C}+\mathrm{O}$ layer exists in the atmosphere of SN 2009dc.

In Figure 4, we show the line velocity of Si II $\lambda 6355$ together with those in other SNe Ia. The Si II line velocity of SN $2009 \mathrm{dc}$ is $\sim 8000 \mathrm{~km} \mathrm{~s}^{-1}$ at -4 days and then decreases to $\sim 6000 \mathrm{~km} \mathrm{~s}^{-1}$ by +24 days. This indicates that SN 2009dc is one of the most slowly expanding SNe Ia (except for faint SNe Ia). The line velocity is much lower than that of SN $2006 \mathrm{gz}$, while comparable with that of SN $2003 \mathrm{fg}\left(8000 \pm 500 \mathrm{~km} \mathrm{~s}^{-1}\right.$ at +2 days; Howell et al. 2006). The velocity of the $\mathrm{C}$ II $\lambda 6580$ line in $\mathrm{SN}$ 2009 dc evolves roughly as well as that of the Si II line (Fig. 4).

\section{DISCUSSION AND CONCLUSIONS}

We summarize the observational characteristics of the peculiar SN Ia 2009dc as follows: (1) one of the slowest evolution of the light curve, i.e., $\Delta m_{15}(B)=0.65 \pm 0.03$, (2) one of the most luminous SNe Ia, i.e., $M_{V}=-19.90 \pm$ 0.15 or brighter, (3) a strong carbon feature in the early spectra, and (4) the lowest expansion velocity among normal and overluminous SNe Ia. The first three features are similar to another super-Chandrasekhar candidate SN 
2006gz, while the last item is clearly different. Although the detailed data for the distant super-Chandrasekhar candidate SN $2003 \mathrm{fg}$ are lacking, its expansion velocity is comparable to that of SN 2009dc. The C II $\lambda 6580$ feature is still present at +5 days in SN $2009 \mathrm{dc}$, although it diminishes by the similar epoch in SNe 2003fg and 2006gz. These facts suggests an existence of a massive unburned $\mathrm{C}+\mathrm{O}$ layer in the ejecta of SN 2009dc. Additionally, we derive the amount of ejected ${ }^{56} \mathrm{Ni}$ mass of $1.2 \pm 0.3 M_{\odot}$ even for no host extinction. If we assume the host extinction of $E(B-V)=0.14 \mathrm{mag}$ and $R_{V}=2.1$, it reaches
$1.6 \pm 0.4 M_{\odot}$. Therefore, we suggest that SN 2009dc is a SN Ia explosion with a super-Chandrasekhar mass WD.

This research has been supported in part by the Grantin-Aid for Scientific Research from JSPS (20540226, 20740107, 21018007, 20840007) and MEXT (19047004, 20040004), and WPI Initiative, MEXT. M.T. has been supported by the JSPS Research Fellowship for Young Scientists.

\section{REFERENCES}

Arnett, W. D. 1982, ApJ, 253, 785

Altavilla, G., et al. 2004, MNRAS, 349, 1344

Benetti, S., et al. 2004, MNRAS, 348, 261

Branch, D., Fisher, A., \& Nugent, P. 1993, AJ, 106, 2383

Conley, A., et al. 2006, AJ, 132, 1707

Elias-Rosa, N., et al. 2006, MNRAS, 369, 1880

Falco, E. E., et al. 1999, PASP, 111, 438

Filippenko, A. V., et al., 1992, AJ, 104, 1543

Filippenko, A. V. 1997, ARA\&A, 35, 309

Garavini, G., et al. 2004, ApJ, 613, 1120

Harutyunyan, A., Elias-Rosa, N. \& Benetti, S. 2009, CBET, 1768 , 1

Hicken, M., Garnavich, P. M., Prieto, J. L., Blondin, S., Depoy, D. L., Kirshner, R. P., \& Parrent, J. 2007, ApJ, 669, L17

Hillebrandt, W., \& Niemeyer, J. C. 2000, ARA\&A, 38, 191

Howell, D. A., et al. 2006, Nature, 443, 308

Kawabata, K. S., et al. 2008, Proc, SPIE, 7014, 10144

Krisciunas, K., Phillips, M. M. \& Suntzeff, N. B. 2004, ApJ, 602, L81

Krisciunas, K., Prieto, J. L., Garnavich, P. M., Riley, J.-L. G., Rest, A., Stubbs, C., \& McMillan, R. 2006, AJ, 131, 1639

Landolt, A. U. 1992, AJ, 104, 340

Li, W., Filippenko, A. V., Treffers, R. R., Riess, A. G., Hu, J., \& Qiu, Y. 2001, ApJ, 546, 734
Lira, P., et al. 1998, AJ, 115, 453

Nomoto, K., Iwamoto, K., \& Kishimoto, N. 1997, Science, 276. 1378

Pastorello, A., et al. 2007, MNRAS, 377, 1531

Perlmutter, S., et al. 1999, ApJ, 517, 565

Phillips, M. M. 1993, ApJ, 413, L105

Riess, A. G., et al. 1998, AJ, 116, 100

Puckett, T., Moore, R., Newton, J., \& Orff, T. 2009, CBET, 1762

Shlegel, D. J., Finkbeiner, D. P., \& Davis, M. 1998, ApJ, 500, 525

Stanishev, V., et al. 2007, A\&A, 469, 645

Stritzinger, M. \& Leibudgut, B. 2005, A\&A, 431, 423

Tanaka, M., et al. 2008, ApJ, 677, 448

Tanaka, M., et al. 2009, ApJ submitted (arXiv:0908.2057)

Turatto, M., Benetti, S. \& Cappellaro, E. 2003, in From Trilight to Highlight: The Physics of Supernovae, ed. W. Hillebrandt \& B. Leibundgut(Berlin: Springer), 200

Wang, X. F., et al. 2006, ApJ, 645, 488

Wang, X. F., et al. 2009, ApJ, 697, 380

Yamanaka, M., Naito, H., Kinugasa, K.,Takanashi, N., Tanaka, M., Kawabata S. K., Ozaki, S., Narusawa, S. \& Sadakane, K. 2009, PASJ, in press

Yanagisawa, K., et al. 2006, SPIE, 6269, 118 